\title{
ÁRVORE DE DECISÃO E ANÁLISE BASEADA EM OBJETOS NA CLASSIFICAÇÃO DE IMAGENS COM RESOLUÇÃO ESPACIAL SUBMÉTRICA ADQUIRIDAS POR VẢNT
}

\section{Object based analysis and decision tree for the classification of submetrical spatial resolution images captured by $U A V$}

\author{
Luis Fernando Chimelo Ruiz ${ }^{1}$ \\ Laurindo Antonio Guasselli 2 \\ Alexandre ten Caten ${ }^{3}$
}

Programa de Pós-Graduação em Sensoriamento Remoto, Universidade Federal do Rio Grande do Sul (UFRGS), CEP: 91509-900, Porto Alegre, Rio Grande do Sul, Brasil

E-mail: ruiz.ch@gmail.com ${ }^{1}$

Universidade Federal do Rio Grande do Sul (UFRGS), CEP: 91509-900, Porto Alegre, Rio Grande do Sul, Brasil

E-mail: laurindo.guasselli@ufrgs.br 2

Universidade Federal de Santa Catarina (UFSC), CEP: 89.520-000, Curitibanos, Santa Catarina, Brasil E-mail: ten.caten@ufsc.br 3

\section{Resumo:}

As imagens com resolução espacial submétrica, como as adquiridas por Veículo Aéreo Não Tripulado (VANT), apresentam alta variabilidade espectral. A análise baseada em objetos possibilita a redução dessa variabilidade e a geração de atributos, aumentando a dimensionalidade do conjunto de dados. Funções de seleção de atributos relevantes e de reforço, disponíveis no algoritmo C5.0, e a análise baseada em objetos facilitam a classificação dessas imagens. Este trabalho teve como objetivos: (i) avaliar as classificações dos objetos em relação aos parâmetros de seleção de atributos (winnow), de reforço (trial) e do Número Mínimo de Amostras (NMA), (ii) determinar os atributos preditivos mais importantes e (iii) comparar a classificação por Árvore de Decisão com Máquina de Vetores Suporte. Para segmentar a imagem foi utilizado o método crescimento de regiões e para a classificação o algoritmo C5.0. Os valores dos parâmetros trial (10) e NMA (5) resultaram acurácias superiores a 0,8. Com esses parâmetros o valor do kappa foi superior a SVM. Ao habilitar o parâmetro winnow foi observado uma redução da dimensionalidade do conjunto de dados de aproximadamente $30 \%$. Os dois atributos mais importantes na discriminação das classes foram a razão entre as bandas verde e azul e a média dos valores das elevações.

Palavras-chave: mineração de dados, mapeamento da cobertura da terra, Veículo Aéreo Não Tripulado (VANT).

\section{Abstract:}

Remotely sensed images acquired with submetrical spatial resolution, such as those captured by Unmanned Aerial Vehicle (UAVs), usually present a high spectral variability. An object based 
approach allows the generation of attributes, increasing the dimensionality of the original dataset. Selection functions for relevant features and booster, available in the C5.0 algorithm, and the objects based analysis facilitate the classification of such datasets. This study aimed at: (i) to evaluate object classification approach in relation to the parameters of attributes selection (winnow), booster (trial) and Minimum Sample Size (MSS), (ii) to determine the most predictive features, and (iii) to compare the classification performance of both Decision Tree and Support Vectors Machine. A region growing method was used in order to segment the image. C5.0 algorithm was used in the classification procedure. The values of the trial (10) and MMS (5) parameters resulted in accuracies higher than 0.8. The kappa value was greater that the SVM, with these parameters. A significant decrease in the data dimensionality up to $30 \%$ was observed when the winnow parameter was habilitated. The two most important features in the breakdown of classes were 'ratio between the blue and green bands' and 'average of the elevation values'.

Keywords: data mining, land cover mapping, Unmanned Aerial Vehicle (UAV).

\section{Introdução}

Entender os padrões e a dinâmica da cobertura da terra é essencial para compreender os efeitos das ações antrópicas na paisagem. Tais efeitos causam alterações locais e globais, sendo o seu entendimento ainda limitado (Jia et al., 2014). Para entender as alterações em escala local dos diversos componentes da paisagem, são necessários dados com resolução espacial métrica ou, se disponível, submétrica (Flener et al., 2013; Saarinen et al., 2013).

Uma alternativa para coletar imagens com alta resolução espacial é o uso de câmaras não métricas acopladas a Veículos Aéreos Não Tripulados (VANT) (Turner et al., 2012). Por outro lado, as imagens com resolução espacial submétrica coletadas por essa tecnologia possuem alta variabilidade espectral, o que dificulta a classificação da cobertura da terra. A redução da variabilidade espectral dessas imagens pode ser obtida a partir da Análise de Imagens Baseada em Objetos (Object-Based Image Analysis - OBIA) (Lechner et al., 2012).

Na OBIA, a unidade de processamento são regiões ou segmentos, que formam objetos com semelhanças espectrais por meio de técnicas de segmentação. A utilização dessa técnica por meio de objetos propicia a geração de atributos geométricos e contextuais. Com isso, há um aumento da dimensionalidade do conjunto de dados (Blaschke, 2010). Esse aumento da dimensionalidade do conjunto de dados impõe mais uma etapa na classificação da cobertura da terra, que está associada tanto à redução da dimensionalidade, quanto à remoção de atributos correlacionados e não informativos (Laliberte et al., 2012).

Laliberte e Rango (2011b) apresentam uma metodologia para classificar pastagens por meio de imagens com resolução espacial submétrica obtidas por um VANT de asa fixa. Essa metodologia combina a segmentação da imagem, a seleção de atributos, utilizando o modelo de classificação e regressão por árvore (Classification And Regression Tree - CART) e a classificação dos objetos pelo método vizinho mais próximo. Aplicando essa metodologia em uma ortoimagem com resolução espacial de $5 \mathrm{~cm}$, os autores classificaram pastagens a nível de espécie com uma acurácia global de $78 \%$, e para nível de grupo de $81 \%$.

Hellesen e Matikainen (2013) integraram OBIA e o algoritmo CART com intuito de diferenciar as classes: floresta, construção e solo (solo sem vegetação e com vegetação), utilizando como entrada dados de Laser Scanner Aerotransportado (LSA) e imagens da câmara UltraCam com resolução espacial de $16 \mathrm{~cm}$. Os autores encontraram valores de acurácias globais de até 96,7\%. 
Uma abordagem semelhante foi utilizada por Hamedianfar et al. (2014) para classificar duas imagens hiperespectrais de áreas urbanas, no qual utilizaram a OBIA com o classificador Árvore de Decisão (AD) C4.5, conseguindo valores de acurácias globais de 93,4\%. Os autores ainda relatam que a $\mathrm{AD}$ aumentou a produtividade da OBIA, acelerou o processo seleção de atributos e resultou em um simples modelo de classificação.

Os modelos por $\mathrm{AD}$ são técnicas de mineração de dados que possibilitam tanto a redução da dimensionalidade dos dados quanto a classificação dos objetos (Hellesen e Matikainen, 2013; Hamedianfar et al., 2014). Além disso, as AD apresentam vantagens em relação a outras técnicas de mineração de dados, como a facilidade de interpretação dos seus resultados e o baixo tempo para a construção do modelo (Friedl e Brodley, 1997). A seleção dos atributos preditivos por meio dessas $\mathrm{AD}$ ocorre no momento da construção do modelo, atributos que não estão nos nós internos da árvore são desconsiderados. Outra possibilidade de selecionar os atributos é pela avaliação da taxa de uso dos seus valores no modelo resultante (Fernandes et al., 2014).

O algoritmo C5.0, sucessor do C4.5, já possui uma função específica para seleção de atributos preditivos mais relevantes e de reforço (boosting). A seleção de atributos é controlada pelo parâmetro winnow, e a função de reforço é controlada pelo parâmetro trial. Diferentes valores para esses parâmetros geram distintas classificações. Devido a isso e às inovações disponíveis no algoritmo C5.0, são necessárias investigações com intuito de avaliar a sua performance na classificação de imagens com resolução espacial submétrica.

Nesse sentido, este trabalho tem como objetivos: (i) avaliar as classificações dos objetos em relação aos parâmetros de seleção de atributos (winnow), de reforço (trial) e do Número Mínimo de Amostras (NMA), contidos no algoritmo C5.0; (ii) determinar os atributos preditivos mais importantes para a discriminação das classes de cobertura da terra e (iii) comparar a classificação por AD com Máquina de Vetores Suporte (Support Vector Machines - SVM).

\section{Metodologia}

A metodologia utilizada está apresentada no fluxograma ilustrado na Figura 1. 


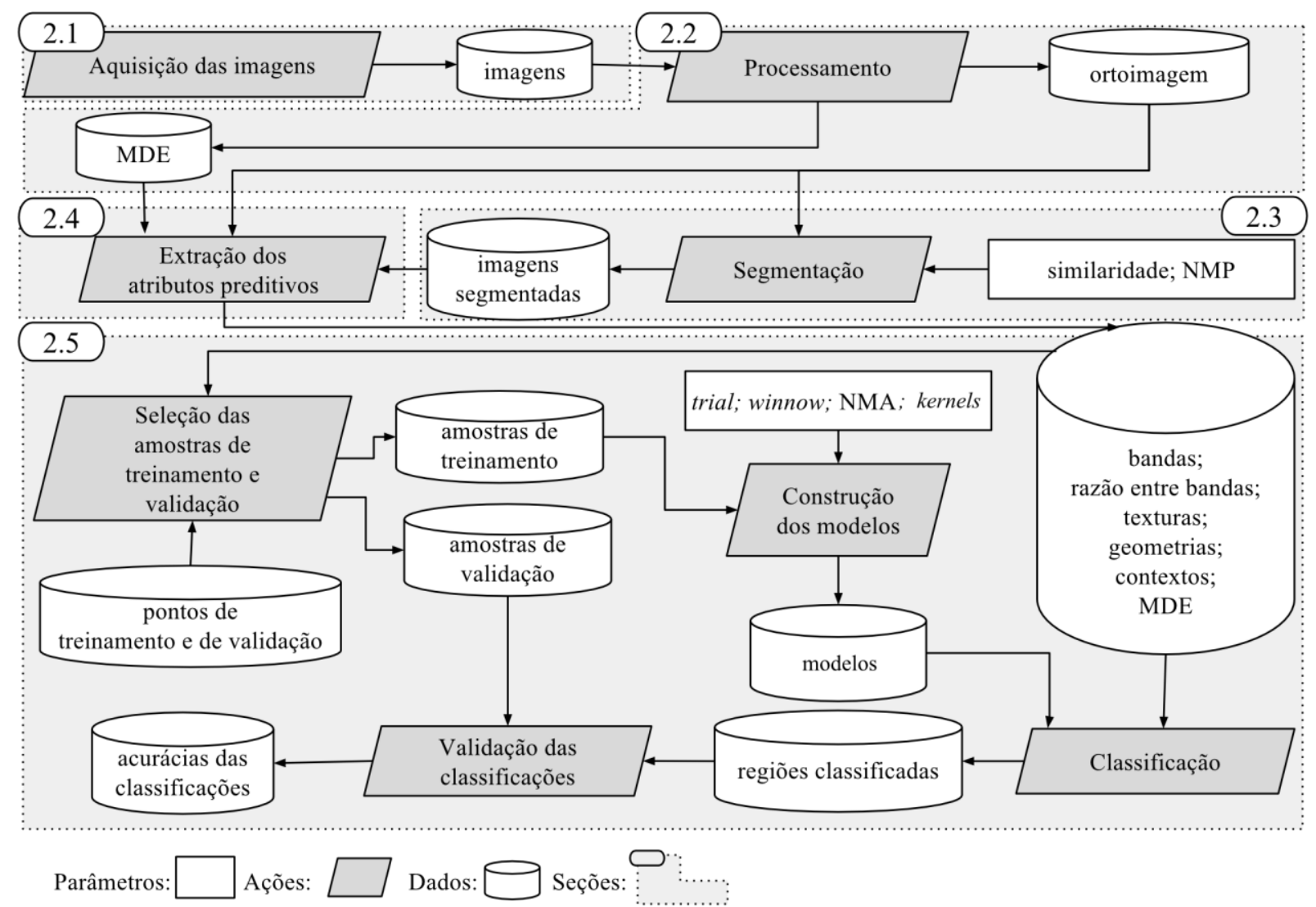

Figura 1: Fluxograma metodológico.

\subsection{Processamento}

Como a câmara é não métrica, os dados de calibração geométrica da câmara foram adquiridos antes do voo pelo programa Agisoft Lens (AgiSoft, 2015). Com essa calibração, são determinados os parâmetros: distância focal, coordenadas do ponto principal e distorções radiais, para a orientação interior das imagens.

Para o processamento das imagens, foi utilizado o programa PhotoScan (AgiSoft, 2015). O PhotoScan possui um fluxo automatizado para processar as imagens, utilizando, para isso, algoritmos de computação visual. Para melhorar o desempenho dos processamentos, o programa utiliza recursos da unidade de processamento (Central Processing Unit - CPU) e da unidade de processamento gráfico (Graphics Processing Unit - GPU). As etapas seguidas no programa foram as seguintes: inserção dos dados auxiliares (parâmetros da orientação interior e dados do GPS); alinhamento das imagens; otimização do alinhamento das imagens; determinação da nuvem de pontos densa; construção da malha triangular; exportação da ortoimagem e do Modelo Digital de Elevação (MDE) (Agisoft, 2015). A ortoimagem foi exportada no sistema de projeção Universal Transversa de Mercator (UTM), fuso 22, sistema de referência SIRGAS2000, com uma resolução espacial de $15 \mathrm{~cm}$. O valor do nível digital de saída foi calculado pela média dos valores sobrepostos. 


\subsection{Segmentação}

Para segmentar a imagem, foi utilizado o método crescimento por regiões, disponível no programa Spring (Câmara et al., 1996). A escolha desse procedimento de segmentação foi baseada em resultados alcançados por Neubert et al. (2006). O método de segmentação de imagens implementada nesse programa necessita de dois parâmetros, um valor para o limiar de similaridade e outro para o Número Mínimo de Pixels (NMP). Os objetos com total de pixels menor que a preestabelecida pelo NMP são agrupados aos seus vizinhos mais semelhantes. Os resultados obtidos por esse método são sensíveis aos valores dos limiares de similaridade e de NMP (Espindola et al., 2006). Dessa forma, foram testados os valores de similaridade 1, 2, 5, 10, 15, 20, 30, 40 e 50. Já para o parâmetro NMP, foram avaliados os valores 20 e 100. Esses valores foram selecionados com base em Teodoro e Araújo (2014).

\subsection{Extração dos atributos preditivos}

Fotointérpretes, ao interpretarem imagens de satélites visualmente, consideram intuitivamente o contexto, os limites, as formas e a variação dos tons de cores para distinguir os objetos. Os atributos preditivos têm como objetivo representar essas informações das imagens e, com isso, facilitar a classificação desses objetos. Na classificação da cobertura da terra utilizando a abordagem baseada em objetos, são usualmente adotados atributos espectrais, texturais, contextuais e geométricos (Blaschke, 2010). Neste trabalho, os atributos preditivos utilizados estão descritos abaixo:

Geométricos: Atributos geométricos são originados da morfologia matemática. Neste trabalho, foram utilizados os seguintes atributos geométricos: compacidade, suavidade, retangularidade, isometria, anisometria, bulkiness, excentricidade, roudness, fator de forma circular e área. Detalhes desses atributos podem ser obtidos no trabalho de Ferreira et al. (2013). Para extrair essas geometrias, foi desenvolvida uma rotina de programação na linguagem Python 2.7 (Rossum, 1995).

Contextual: Atributos de contexto consideram os limites e os valores dos segmentos vizinhos. Neste trabalho, foi adotado como contexto o cálculo da diferença média dos valores das bandas do objeto entre seus vizinhos (Laliberte e Rango, 2011b). Essa diferença média foi calculada para as três bandas espectrais, formando três atributos de contexto, dif_media_ivp (banda do IVP), dif_media_verde (banda do verde) e dif_media_azul (banda do azul). Esse atributo foi extraído por meio de rotina de programação desenvolvida na linguagem Python 2.7 (Rossum, 1995).

Espectrais: O conjunto de dados espectrais é referente aos valores dos Níveis Digitais (ND) da imagem. Neste trabalho, foram utilizados os valores médios e o Desvio Padrão (DP) das bandas do azul, verde e do IVP, formando os atributos media_azul, DP_azul, media_verde, DP_verde, media_ivp e DP_ivp. Por meio das bandas, foram derivadas as razões entre as bandas do IVP com azul (r_ivp_a), razão do IVP com o verde (r_ivp_v) e a razão da banda do verde com a azul (r_v_a). Também foi calculada a divisão do valor de cada objeto pela soma dos valores de cada banda, formando mais três atributos espectrais, razao_azul, razao_verde e razao_ivp. Esses atributos espectrais foram selecionados conforme Laliberte e Rango (2011b).

Texturais: A textura da imagem pode ser definida como o padrão das variações de brilho ou de níveis de cinza dentro de uma imagem ou dentro de uma região da imagem (Karathanassi, 2000). 
Neste trabalho, foi utilizado o método estatístico de segunda ordem, que utiliza a matriz de coocorrência dos níveis de cinza (Grey Level Coocurrence Matriz - GLCM) para a extração das texturas. A partir da GLCM, foram extraídas as 13 características de texturas definidas por Haralick (1973). Essas texturas foram extraídas a partir da ferramenta r.texture do programa GRASS-GIS 6.4 (GRASS Development Team, 2015) para a média das três bandas da ortoimagem. A extração da textura para os objetos da segmentação foi baseada no estudo de Laliberte e Rango (2009). Foi utilizada uma janela de análise no tamanho 3x3 e, para orientação, foram utilizados os ângulos $0,45,90$ e 135. O valor de textura final, foi obtido da média dos quatro valores de ângulo de análise.

Modelo Digital de Elevação (MDE): O MDE foi obtido pela interpolação da nuvem de pontos cotados, formando os atributos preditivos com valores médios (media_elev) e o desvio padrão das elevações dos objetos (DP_elev). A nuvem de pontos foi gerada no programa PhotoScan e interpolada utilizando redes de triângulos irregulares com função de primeira ordem. A área de estudo possui um desnível de aproximadamente 18 metros, considerando as medidas sobre o terreno. Os cálculos e a determinação das médias e dos desvios padrão dos atributos espectrais, texturais e MDE, foram calculados para cada objeto da segmentação, utilizando um algoritmo desenvolvido em Python 2.7 (Rossum, 1995).

\subsection{Classificação e validação}

As amostras de treinamento para cada segmentação foram obtidas utilizando pontos regulares. Esses pontos foram gerados com equidistância de 25 metros, gerando um total de 1155 pontos de forma regular sobre toda a área de estudo e compreendendo todas as classes de cobertura da terra, que neste estudo foram: mata nativa (MN), silvicultura (SV), sombra (SO), pastagem (PA), resteva (RE), construção (CO), solo exposto (SE), campo (CA), banhado (BA), macrófita (MA), água/cinza (AC), água ( $\mathrm{AG}$ ) e solo úmido (SU). Para validar as classificações, foram gerados 500 pontos aleatórios independentes dos dados de treinamento. A atribuição das classes desses pontos ocorreu por meio da interpretação da ortoimagem. Após a geração das amostras de validação, foi calculado o índice kappa. A primeira etapa para gerar as amostras de treinamento e de validação foi amostrar os objetos de cada segmentação, obtendo um arquivo no formato shapefile que possui, na tabela de atributos, um identificador para a classe de cobertura da terra e outro para os objetos da segmentação. O identificador dos objetos possibilita integrar os atributos preditivos, gerando as amostras de treinamento e de validação.

Para construir os modelos por AD, foi utilizada a biblioteca de programação C5.0 disponível na linguagem R (R Core Team, 2014). As AD podem ser entendidas como uma hierarquia de nós que são conectados por ramos, sua estrutura se iguala a uma árvore invertida, desenvolvendo-se da raiz para a folha. O nó interno (nó de decisão) possui o teste de condição para algum atributo, e cada ramo descendente corresponde a um possível valor para esse atributo, sendo a folha da árvore relacionada a uma classe. O caminho completo da árvore, que vai da raiz até a folha, determina uma regra de classificação (Quinlan, 1996).

No algoritmo C5.0, a função de reforço (boosting) tem como finalidade gerar vários modelos, também chamados de ensaios, ao invés de apenas um modelo. O parâmetro trial controla o número de vezes que serão gerados os ensaios. Para cada construção do modelo é dada mais atenção às regras de classificação com maiores taxas de erros, tentando melhorá-las no próximo ensaio (Quinlan, 2013). 
Outra inovação do algoritmo C5.0 é a possibilidade de selecionar atributos preditivos mais informativos, controlado pelo parâmetro winnow. Essa seleção inicia dividindo aleatoriamente em duas partes iguais os dados de treinamento, posteriormente, com uma dessas partes é gerado o modelo por árvore. Esse modelo tem como propósito avaliar a utilidade dos atributos preditivos. Tais atributos, que não estão em nenhuma divisão da árvore, são considerados sem importância e podem ser removidos do conjunto de dados. A outra parte das amostras de treinamento, que não foi utilizada para gerar o modelo, é empregada para estimar a taxa de erro do modelo. A taxa de erro é calculada com e sem o atributo preditivo. Caso essa taxa aumente com a inserção do atributo preditivo, sua remoção do conjunto de dados é realizada (Kuhn e Johnson, 2013).

Foram testados os valores $0,5,10,20,30,40,50,60,70,80,90$ e 100 para o parâmetro trial, quanto maior esse valor, mais iterações (ensaios) são feitas. Também foram avaliados os valores 2, 5, 10, 20, 30, 40 e 50 para o parâmetro NMA, conforme Ruiz et al. (2014). Quanto maior o valor do NMA, maior o número de amostras analisadas nos nós internos da árvore, e consequentemente, menores são as árvores resultantes. O parâmetro winnow é controlado por valores boleanos, True ou False, essas para cada modelo gerado foram testados os dois valores.

As classificações realizadas com SVM foram avaliadas para os kernels polinomiais e de Funções de Base Radial (FBR). As funções de kernel têm como a finalidade transformar um problema não linearmente separável (espaço de entrada) em um problema linearmente separável (espaço das características). Para o kernel polinomial foram avaliados valores de ordem de 1 a 8 , já para o kernel de FBR, os valores de sigmas foram de 1 a 20, conforme avaliado por Huang et al. (2002). Esses modelos foram gerados por meio da linguagem Python e da biblioteca de programação Scikit-Learn.

Por meio das amostras de validação formadas com base nos 500 pontos aleatórios, foi calculado para cada classificação o índice kappa, para isso se utilizou a biblioteca StatsModels. O índice kappa é uma técnica discreta multivariada utilizada na determinação da acurácia de mapas temáticos ( $\mathrm{Gu}$ et al., 2015). Também foi utilizada uma classificação qualitativa dos valores de kappa, conforme o trabalho de Landis e Koch (1977). A segmentação que resultou no menor Número Total de Objetos (NTO) e no modelo de AD com o maior kappa foi utilizada para avaliar o comportamento dos parâmetros do classificador C5.0 e para gerar as classificações por SVM. Valores baixos para o NTO e altos para o kappa indicam que a segmentação possibilitou uma representação confiável das classes de cobertura da terra. Considerando a segmentação, os parâmetros do algoritmo C5.0 e as classificações por SVM, o modelo de classificação com maior valor de kappa de cada algoritmo foi utilizada para avaliar o erro de classificação em relação às classes de cobertura da terra. Esse erro de classificação por classe foi calculado por meio da Acurácia do Produtor (AP) (Gu et al., 2015).

\section{Resultados e discussões}

Os resultados das segmentações e dos parâmetros do classificador C5.0 são apresentados na Tabela 1, de acordo com a qualidade do Kappa. Destaca-se que os valores de limiares 30, 40 e 50 foram descartados, porque os objetos gerados por esses limiares continham mais que uma classe de cobertura da terra. Ou seja, objetos gerados não tiveram capacidade suficiente para discriminar as classes de cobertura da terra, fazendo com que os objetos possuíssem duas classes para o mesmo segmento. 
Conforme a Tabela 1, a segmentação com limiar de similaridade igual a 10 e o NMP igual a 100 se mostrou mais apropriada, uma vez que seu valor de kappa foi de 0,83 e o NTO permaneceu entre os menores valores. A segmentação com similaridade igual a 15 e NMP igual a 20 resultou em um kappa igual ao supracitado, no entanto, o NTO foi aproximadamente quatro vezes maior, com isso o número de objetos classificados erroneamente foi maior.

Teodoro e Araújo (2014), ao avaliar a segmentação por crescimento de regiões, relatam que os valores baixos para a similaridade (1,2 ou 5) e para o NMP (9), assim como valores altos para ambos os parâmetros (50 similaridade e 100 NMP), resultaram nos piores valores de kappa. Segundo os autores, combinando os valores baixos, foram gerados muitos objetos semelhantes, sendo alguns quase impossíveis de serem discriminados. Para combinações com valores altos, pixels distintos foram agrupados no mesmo objeto, não podendo mais serem separados, diminuindo, assim, a acurácia da classificação. Neste estudo, as melhores combinações foram para valores de similaridade 10 ou 15 e NMP mais elevado, igual a 100.

Tabela 1: Segmentações e parâmetros do classificador C5.0 que resultaram nos valores de kappa mais altos

\begin{tabular}{cccccccc}
\hline Limiar & NMP & NTO & Winnow & Trial & NMA & Kappa & $\begin{array}{c}\text { Qualitativo } \\
\text { (Landis e } \\
\text { Koch, 1977) }\end{array}$ \\
\hline 1 & 20 & 696777 & True & 10 & 5 & 0.75 & Muito boa \\
\hline 2 & 20 & 688503 & True & 100 & 2 & 0.77 & Muito boa \\
\hline 5 & 20 & 630417 & True & 100 & 5 & 0.73 & Muito boa \\
\hline 10 & 20 & 433488 & False & 10 & 5 & 0.79 & Muito boa \\
\hline 15 & 20 & 283618 & True & 60 & 2 & 0.83 & Excelente \\
\hline 20 & 20 & 185431 & False & 80 & 2 & 0.78 & Muito boa \\
\hline 1 & 100 & 112873 & True & 20 & 2 & 0.73 & Muito boa \\
\hline 2 & 100 & 111565 & False & 80 & 5 & 0.75 & Muito boa \\
\hline 5 & 100 & 105755 & False & 80 & 5 & 0.79 & Muito boa \\
\hline 10 & 100 & 76825 & False & 80 & 5 & 0.83 & Excelente \\
\hline 15 & 100 & 51603 & False & 90 & 5 & 0.82 & Excelente \\
\hline 20 & 100 & 33196 & False & 10 & 2 & 0.81 & Excelente \\
\hline
\end{tabular}

Ao analisar o parâmetro NMA, na Tabela 1, foram encontrados os valores 2 e 5. Tais valores possibilitam um maior crescimento da $\mathrm{AD}$, consequentemente, são geradas árvores com um menor poder de generalização. Neste trabalho, ocorreu o contrário, pois baixos valores para o NMA resultaram em altos valores de índice kappa. Isso pode ser explicado devido às amostras de treinamento serem coletadas de forma regular sobre toda área de estudo, possibilitando a captura das particularidades das classes que resultaram em modelos com alto poder de generalização.

Com intuito de avaliar o comportamento dos parâmetros da AD C5.0, foi selecionada a segmentação que resultou, concomitantemente, no menor NTO e no maior valor do kappa, neste caso, foi a segmentação com limiar de similaridade igual a 10 e NMP igual a 100. A Figura 2 ilustra o comportamento dos modelos por árvore de decisão gerados para essa segmentação.

Analisando a Figura 2a, pode-se identificar a estabilização da acurácia dos modelos, quando o parâmetro trial recebe o valor 10. Isso significa que, construindo 10 ensaios e diminuindo o erro 
de classificação sobre as regras com maiores taxas de erros, foi possível construir modelos com um alto poder de predição.

Há em média um aumento de $9 \%$ da acurácia para os modelos construídos com trial igual a 10 em relação aos gerados com trial igual a 0. Quinlan (2013) relata que em média o valor 10 para o trial possibilita uma redução de $25 \%$ na taxa erro do modelo. Neste estudo, o valor 10 para o parâmetro trial e a utilização de amostras de treinamento coletadas de forma regular proporcionaram resultados considerados Excelentes, conforme Landis e Koch (1977).

Observa-se que o NMA igual a 2, 5 e 10 possibilitou os maiores valores do kappa, e que os valores 2 e 5 se sobressaem (Figura $2 \mathrm{a}$ ). O número de folhas que representa o tamanho da árvore de decisão também pode ser atribuído ao parâmetro NMA. Para os valores 2, 5, 10, 20, 30, 40 e 50, foram encontrados os valores médios: 99, 60, 34, 16, 13, 10 e 8, respectivamente. Para este estudo, o valor 5 foi o que apresentou, simultaneamente, o maior valor de kappa e o menor número de folhas. $\mathrm{O}$ número de folhas reduzido facilita a interpretação do modelo. Para os modelos que utilizaram True para o parâmetro winnow o comportamento foi semelhante, como pode ser observado na Figura $2 b$, em que as diferenças dos valores kappa entre winnow igual a True e igual a False, no geral, resultaram em valores baixos, permanecendo entre o intervalo 0,03 a 0,03 . A Figura $2 \mathrm{~b}$ também mostra que os modelos com winnow igual a True resultaram em maiores valores para o índice kappa, dado que a maior parte dos valores estão acima do zero, como pode ser identificado na Figura 2 b. Os valores obtidos neste estudo estão de acordo com os utilizados na literatura. Powers et al.(2015) estabeleceram em seu estudo o valor 10 para o parâmetro trial e True para o winnow. Os autores obtiveram uma acurácia global de $88 \%$ para a classificação de conflitos industriais na floresta boreal de Alberta, Canadá.

Outro fator importante verificado, quando selecionado True para o parâmetro winnow, foi a redução do número de atributos. Na Figura 2c, observa-se uma redução maior que $30 \%$ para os modelos com valores de NMA iguais a 2, 5 e 10, sem perdas significativas nos valores do índice kappa (Figura 2b). Esses valores aproximam-se aos obtidos por Laliberte e Rango (2011), que identificaram uma redução de $45 \%$ no total de atributos preditivos, utilizando, para isso, o modelo CART. Essa redução do número de atributos na construção dos modelos facilita a classificação da imagem, porque diminui a complexidade do modelo e utiliza menos arquivos na memória RAM (Random Access Memory) do computador.

Ao selecionar True para o parâmetro winnow, o modelo gerado retorna a importância dos atributos preditivos na sua construção para a classificação da segmentação do limiar de similaridade igual a 10, NMP igual a 100, trial igual a 80 e NMP igual a 5. Os atributos com maior importância foram: (i) r_v_a (45\%), (ii) media_elev (13\%), (iii) media_verde (13\%), (iv) razao_verde (8\%), (v) dif_media_ivp (7\%), (vi) area_seg (4\%), (vii) density (3\%), (viii) media_ivp (3\%), (ix) DP_verde $(2 \%)$, (x) media_azul (2\%), (xi) anisometria (2\%), (xii) razao_azul $(2 \%)$ e outros 10 atributos, com importância menor que $1 \%$. Dentre os atributos mais importantes, pode-se destacar os atributos espectrais, a elevação do terreno e os geométricos. Os atributos texturais não foram importantes na discriminação das classes, considerando esse modelo de classificação. 


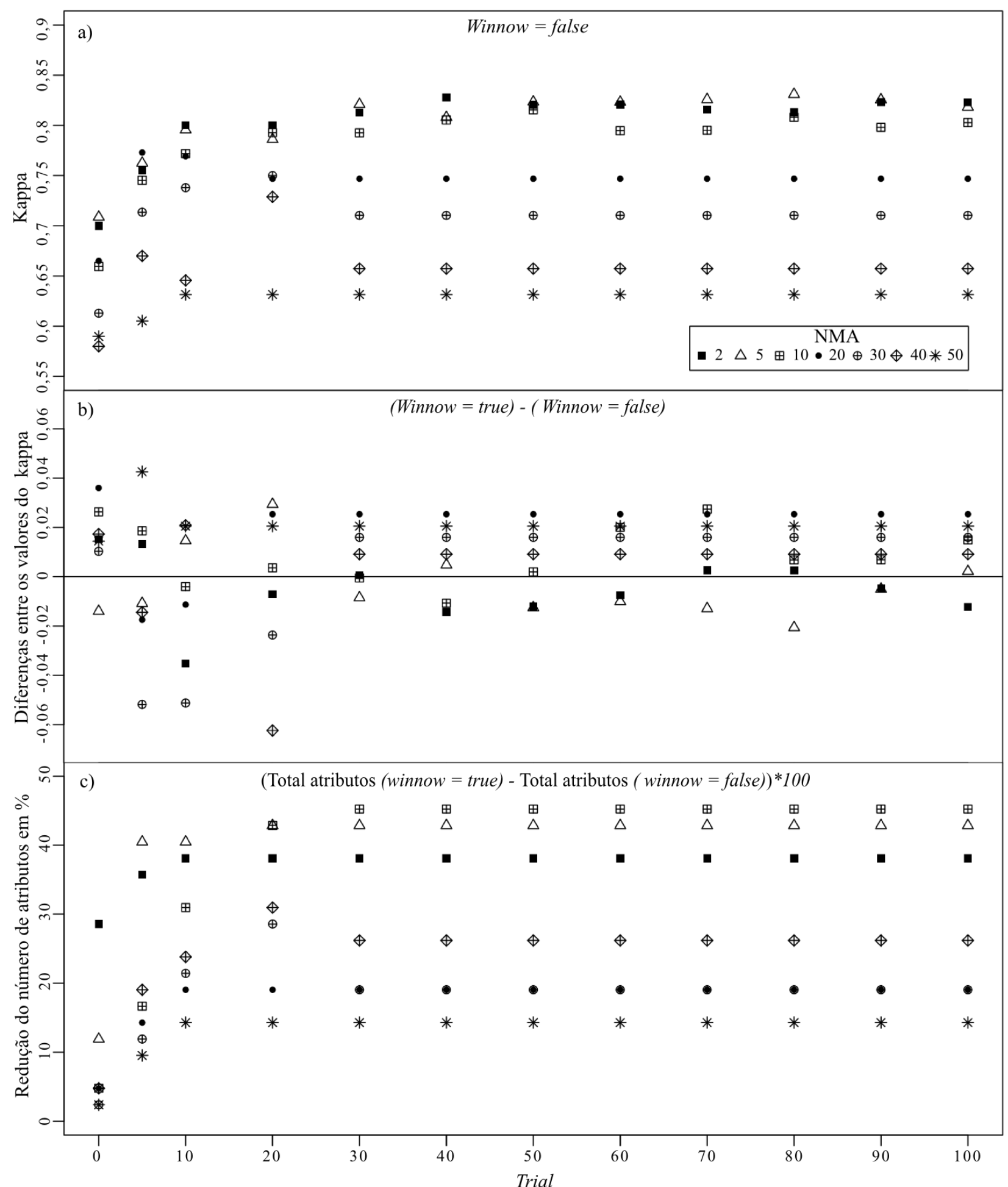

Figura 2: Comportamento dos parâmetros do modelo C5.0 para a segmentação limiar de similaridade igual a 10 e MNP igual a 100: a) modelos com winnow igual a False; b) diferença dos kappas entre os modelos winnow igual a True e igual a False; c) redução do número de atributos comparando winnow igual a True e igual a False.

O atributo mais importante para esse modelo foi a r_v_a. Esse atributo preditivo também foi selecionado como raiz da árvore, pois é o que apresentou o menor valor de impureza, ou seja, o atributo mais significativo para separação das classes. O media_elev pode ter sido selecionado como um dos atributos mais importantes devido à ocorrência das classes silvicultura, mata 
nativa, resteva, água/cinza e solo úmido em locais específicos na área de estudo, possibilitando que o modelo capturasse padrões dessas classes em relação à sua elevação.

Esperava-se que a banda do IVP resultasse em um valor de importância maior que o apresentado, uma vez que as classes de cobertura da terra são na sua maioria vegetação. Supõe-se que isso não ocorreu devido à largura da faixa espectral definida pelo intervalo do filtro inserido na câmara, apresentando sensibilidade em distintos comprimentos de onda e assim, diminuindo o contraste entre os alvos.

Essa suposição é certificada ao averiguar outras câmaras comerciais, como é o caso da Tetracam Mini-MCA6, com seis filtros em intervalos de comprimentos de ondas mais estreitos nas seguintes bandas espectrais: azul $(450 \mathrm{~nm})$, verde $(530 \mathrm{~nm})$, vermelho $(670$ e $700 \mathrm{~nm})$, borda do vermelho $(740 \mathrm{~nm})$ e IVP $(780 \mathrm{~nm})$, esses valores variam $10 \mathrm{~nm}$ do comprimento de onda central. Torres-Sánchez et al. (2013), com objetivo de discriminar ervas daninhas na agricultura, utilizaram essa câmara acoplada a um VANT. Os autores observaram que a maior separabilidade entre vegetação e solo exposto foi dada por meio da razão normalizada entre a banda do vermelho e do IVP.

Para melhor entender as confusões entre as classes, foi avaliada a classificação com o melhor valor de índice kappa. Nesse contexto, o modelo treinado com as amostras do limiar de similaridade igual a 10, NMP igual a 100, trial igual a 80, winnow igual a falso e NMA igual a 5 resultou em um índice kappa de 0,83. Já para a classificação com SVM, o melhor valor de índice kappa foi obtido utilizando o kernel polinomial de primeira ordem, resultando em um valor de kappa igual a 0,77 .

$\mathrm{Na}$ literatura são reportados trabalhos com valores de acurácias semelhantes ao encontrado neste trabalho, como foi o caso do trabalho efetuado por Laliberte et al. (2011a), em que os autores avaliaram a separação de oito classes da cobertura da terra, utilizando uma câmara Mini-MCA6, com seis bandas, obtendo um valor de $87 \%$ para a acurácia global e 0,83 para o índice kappa. Deng et al. (2015) também encontraram acurácias maiores para o algoritmo C5.0 em relação ao SVM na classificação de áreas urbanas com dados de radar de abertura sintética. Os autores também citam que o algoritmo C5.0 foi mais eficiente no consumo de tempo, na seleção dos atributos preditivos e na construção do modelo. Hao et al. (2015) avaliaram os algoritmos Random Forest, SVM e o C5.0 para a classificação de lavouras no norte da China. Eles observaram que, quando os classificadores receberam 4,000 amostras de treinamento, os algoritmos resultaram em desempenhos semelhantes.

A Tabela 2 apresenta as classes de cobertura da terra com os valores da AP calculados a partir das 500 amostras de validação, o número de folhas e o número de amostras de treinamento. Os menores valores de acerto foram encontrados para as classes construção (CO) e macrófita (MA), que resultaram nos valores $0 \%$ e $37,5 \%$, respectivamente. Estes baixos valores para essas classes podem estar relacionados com o número de amostras de treinamento, que, neste caso, não foi suficiente para discriminar essas classes das demais. As classes restantes obtiveram acertos maiores que $68 \%$, em que as classes água/cinza (AC) e água (AG) resultaram em acertos de $100 \%$, cada uma com apenas uma folha de classificação.

A análise do número de folhas e da AP não mostrou qualquer correspondência, pois ocorreram acertos de $100 \%$ e $0 \%$ para o mesmo número de folhas. Entretanto, foi constada uma relação do número de folhas com as amostras de treinamento. Como no caso das classes campo (CA), banhado (BA) e pastagem (PA), que possuem uma maior representatividade na área de estudo, receberam um maior número de amostras de treinamento, consequentemente, o modelo por árvore de decisão gerou um número maior de folhas para capturar as particularidades dessas classes, possibilitando classificá-las com acertos acima de 68\%. Essa representatividade das 
classes em proporções que simulam a sua ocorrência na área de estudo foi possível devido às amostras de treinamento serem coletadas de forma regular sobre toda a área.

Os valores de AP obtidos pelo algoritmo SVM (Tabela 2), na sua maioria, foram menores ou iguais aos obtidos por $\mathrm{AD}$. Esses resultados concordam com os kappas obtidos pelos métodos, uma vez que a AD resultou em um kappa de 0,83 e o SVM em um kappa igual a 0,77.

Tabela 2: AP e o número de folhas do modelo treinado com a segmentação utilizando limiar de similaridade igual 10, NMP igual a 100, winnow igual a falso, trial igual a 80 e NMA igual a 5, AP obtido por SVM e o número de amostras de treinamento

\begin{tabular}{cccccccccccccc}
\hline classes & MN & SV & SO & PA & RE & CO & SE & CA & BA & MA & AC & AG & SU \\
\hline AP (\%)- AD & 75 & 88.8 & 88.8 & 94.6 & 74.2 & 0 & 73.3 & 68 & 90 & 37.5 & 100 & 100 & 89 \\
\hline folhas & 8 & 2 & 3 & 11 & 4 & 1 & 5 & 11 & 9 & 3 & 1 & 1 & 5 \\
\hline AP (\%) -SVM & 81.3 & 55.6 & 88.8 & 91.6 & 81.1 & 33.3 & 66.7 & 78.2 & 79.8 & 37.5 & 100 & 100 & 57.9 \\
\hline treinamento & 54 & 19 & 38 & 400 & 59 & 4 & 19 & 270 & 192 & 19 & 5 & 18 & 58 \\
\hline
\end{tabular}

O número de folhas também exprime a complexidade em separar as classes de cobertura da terra, classes com padrões particulares facilitam a classificação. Essas questões foram analisadas por Ruiz et al. (2014), que utilizaram o mesmo número de amostras de treinamento para cada classe e obtiveram diferentes números de folhas. Nesse trabalho, os autores relatam que, para classificar as classes água e solo exposto, foi necessária apenas uma folha, já para uma classe mais complexa, como floresta, foram necessárias nove folhas.

Em imagens coletadas com VANT (Figura 3a) essa complexidade está associada à resolução espacial submétrica, resultando em uma alta variabilidade espectral. Essa complexidade pode ser agravada pelo número de classes a ser classificada, bem como pela data, hora e pelo intervalo de captura das imagens. Imagens coletadas em intervalos muitos distintos acarretam em diferenças na iluminação, que atribuem para a mesma classe distintos valores de radiância. As áreas classificadas como sombra (SO) (Figura 3b) exprimem a combinação do mês da aquisição com a latitude da área de estudo. Isso promoveu menores ângulos de iluminação para essa região, resultando em sombras mais prolongadas (Figura 3b).

Além disso, algumas classes apresentam um comportamento heterogêneo, como é caso da classe campo (CA), localizada ao norte da Figura 3a, em torno do corpo d'água com sedimentos (água/cinza). Nesse local, essa classe possui uma textura mais rugosa, diferente da encontrada próxima da classe solo úmido (SU) (Figura 3a). Esse comportamento diferenciado dificultou a classificação da classe campo, como pode ser observado na Figura $3 b$, confundindo com pastagem e solo úmido. Esse mesmo comportamento foi identificado para a classe macrófita (MA), apresentando diferenças de vigor vegetativo, que muda conforme sua localização no corpo d'água. Essa diferença, aliada ao número de amostras, pode ter sido responsável pelo baixo valor da AP obtido para essa classe. A classe pastagem (PA) também demonstrou diferenças de vigor vegetativo, como na densidade do dossel. Essas características variaram conforme a época de plantio da cultura nas parcelas. Apesar disso, a pastagem resultou em uma boa classificação, com AP de $94,6 \%$.

Outra questão são as classes que possuem respostas espectrais semelhantes, como foi identificado para as classes água/cinza e construção, mas que, neste estudo, foram separadas por meio dos atributos geométricos: area_seg e isometria, como o contextual: dif_media_ivp. Fernandes et al. (2014) também identificaram a área do objeto (area_seg) como atributo importante para identificar a espécie junco gigante. As classes mata nativa e silvicultura foram discriminadas utilizando os atributos do terreno: DP_elev e media_elev. 

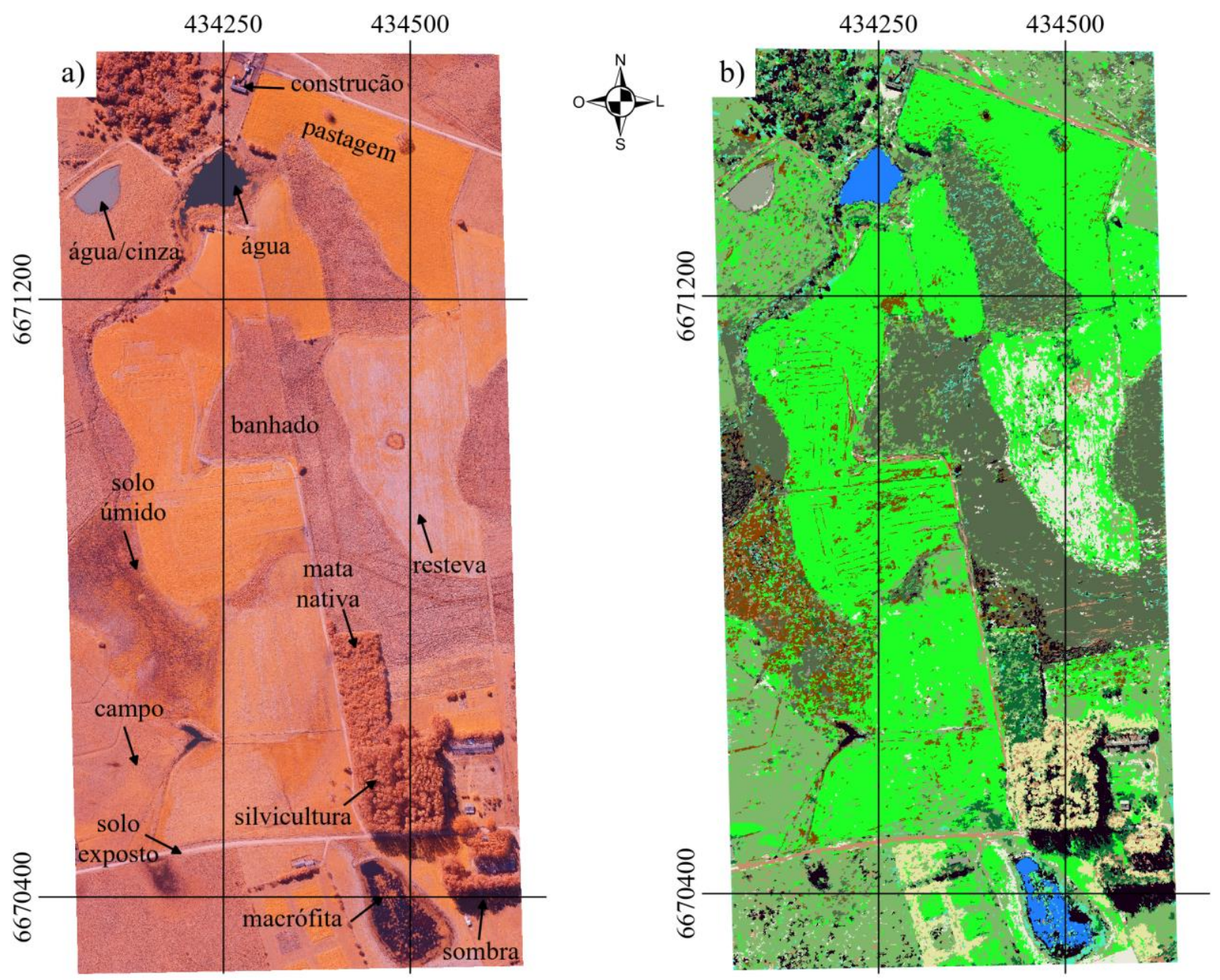

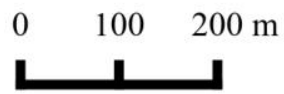

Sistema de Projeção: UTM Fuso: 22

Hemisfério: Sul

Datum horizontal: SIRGAS2000

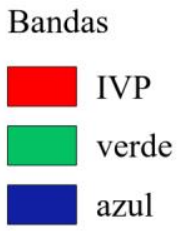

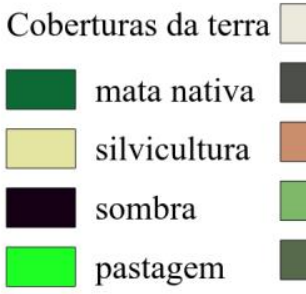

resteva construção solo exposto campo banhado

Figura 3: a) ortoimagem na composiçao: IVP-Vermelho, verde-Verde e azul-Azul; b) classificação da ortoimagem por meio do modelo treinado com amostras do limiar de similaridade igual a 10, NMP igual a 100, trial igual a 80, winnow igual a falso e NMA igual a 5.

\section{Conclusões}

Os maiores valores de kappa e os menores NTO foram encontrados para a segmentação com limiar de similaridade igual a 10 e o NMP igual a 100. Considerando a classificação treinada com as amostras dessa segmentação, foi identificado que o valor 10 para o parâmetro trial e 5 para NMA, são valores indicados para a classificação de imagens com resolução espacial submétrica. Já para o parâmetro winnow, o valor True, foi o mais adequado, devido a redução da dimensionalidade do conjunto de dados e da complexidade do modelo. Neste estudo, a redução 
do conjunto de dados alcançou $30 \%$. A parte mais complexa desta metodologia foi validar todas as classificações derivadas dos vários parâmetros e valores.

Futuros trabalhos podem utilizar os valores dos parâmetros obtidos neste trabalho como guia. Outra sugestão é fixar amostras de treinamento para o parâmetro winnow e não permitir que em cada classificação esse parâmetro selecione amostras de treinamento aleatoriamente. Com isso, em todas as classificações avaliadas, a importância dos atributos preditivos será calculada sobre as mesmas amostras.

Os atributos preditivos mais importantes para a discriminação das classes, foram os seguintes: r_v_a, media_elev, media_verde, razao_verde, dif_media_ivp, area_seg, density, media_ivp, DP_verde, media_azul, anisometria, razao_azul. Futuros trabalhos podem avaliar atributos preditivos derivados do MDE para a classificação.

A integração da OBIA e do classificador C5.0 se mostrou promissora para a classificação de imagens com resolução espacial submétrica. Essa integração tornou possível reduzir a variabilidade espectral da ortoimagem, derivar atributos contextuais e geométricos, selecionar os atributos preditivos mais informativos e classificar a imagem com $87 \%$ de acurácia global e 0,83 para o índice kappa, valores superiores quando comparados à classificação por SVM.

\section{AGRADECIMENTOS}

À Coordenação de Aperfeiçoamento de Pessoal de Nível Superior (CAPES) pela bolsa de estudo em nível de pós-graduação e pelo financiamento do Veículo Aéreo Não Tripulado, Echar 20A. Ao CINDACTA II pela liberação do espaço aéreo (NOTAM E1787/2014; Data: 21/05/2014).

\section{REFERÊNCIAS BIBLIOGRÁFICAS}

Agisoft, Guide Photoscan, 2015. Disponível em: <http://www.agisoft.com/support/tutorials/intermediate-level/>. Acessado em 20 set. 2014.

Blaschke, T. Object based image analysis for remote sensing. ISPRS Journal of Photogrammetry and Remote Sensing, vol. 65, n. 1, p. 2-16, 2010.

Câmara G.; Souza R. C.M.; Freitas,U. M.; Garrido, J. SPRING: "Integrating remote sensingand GIS by object-oriented data modelling". Computers and Graphics, vol. 20, n.3, p. 395-403, 1996.

Chutia, D.; Bhattacharyya D. K.; Kalita, R.; Sudhakar, S. OBCsvmFS: Object-Based Classification supported by Support Vector Machine Feature Selection approach for hyperspectral data. Journal Geomatics, vol. 8, n. 1, p.12-19, 2014.

Deng, L.; Yan, Y.-N.; Sun, C. Use of Sub-Aperture Decomposition for Supervised PolSAR Classification in Urban Area. Remote Sens. n.7, p.1380-1396, 2015.

Espindola, G.; Câmara, G.; Reis, I. A.; Bins, L. S.; Monteiro, A. M. Parameter selection for region-growing image segmentation algorithms using spatial autocorrelation. International Journal of Remote Sensing. vol. 27, n. 14, p. 3035-3040, 2006.

Fernandes, M. R.; Aguiar, F. C.; Silva, J. M. N.; Ferreira, M. T.; Pereira, J. M.C. Optimal attributes for the object based detection of giant reed in riparian habitats: A comparative study 
between airborne high spatial resolution and WorldView-2 imagery. International Journal of Applied Earth Observation and Geoinformation, vol. 32, p. 79 - 91, 2014.

Ferreira, R. S.; Costa, G. A. O. P.; Feitosa, R. Q. Avaliação de critérios de heterogeneidade baseados em atributos morfológicos para segmentação de imagens por crescimento de regiões. Boletim de Ciências Geodésicas, vol. 19, n. 3, p. 452-471, 2013.

Flener, C.; Vaaja, M.; Jaakkola, A.; Krooks, A.; Kaartinen, H.; Kukko, A.; Kasvi, E.; Hyyppä, H.;

Hyyppä, J.; Alho, P. Seamless mapping of river channels at high resolution using mobile LIDAR and UAV-Photography. Remote Sensing, vol.5, n.12, p. 6382-6407. 2013.

Friedl, M.A.; Brodley, C.E. Decision tree classification of land cover from remotely sensed data. Remote Sensing of Environment, vol.61, n. 3, p. 399-409, 1997.

GRASS Development Team, 2015. Geographic Resources Analysis Support System (GRASS) Software, Version 6.4. Open Source Geospatial Foundation. http://grass.osgeo.org.

Gu, J.; Congalton, R. G.; Pan, Y. The Impact of Positional Errors on Soft Classification Accuracy Assessment: A Simulation Analysis. Remote Sensing, n.7, p. 579-599, 2015.

Hamedianfar, A.; Shafri, H. Z. M.; Mansor, S.; Ahmad, N. Combining data mining algorithm and object-based image analysis for detailed urban mapping of hyperspectral images. J. Appl. Remote Sens, vol. 8, n. 1, p. 85-91, 2014.

Hao P, Wang L, Niu Z. Comparison of Hybrid Classifiers for Crop Classification Using Normalized Difference Vegetation Index Time Series: A Case Study for Major Crops in North Xinjiang, China. PLoS ONE, 10(9), 2015.

Haralick, R.M.; Shanmugan, K.; Dinstein, I. Textural features for image classification, IEEE Transactions on Systems, Man, and Cybernetics, vol. 3, p. 610-621, 1973.

Hellesen, T.; Matikainen, L. An object-based approach for mapping shrub and tree cover on grassland habitats by use of LIDAR and CIR orthoimages. Remote Sensing. vol. 5, n. 2, p. 558583, 2013.

Heumann, B.W. An Object-Based Classification of Mangroves Using a Hybrid Decision TreeSupport Vector Machine Approach. Remote Sensing, vol. 3, n. 11, p. 2440-2460, 2011.

Jia, K.; Liang, S.; Wei, X.; Yao, Y.; Su, Y.; Jiang, B.; Wang, X. Land cover classification of Landsat data with phenological features extracted from time series modis NDVI data. Remote Sensing, vol. 6, n. 11, p. 11518-11532, 2014.

Huang, C., Davis, L. S.; Townshend, J. R. G., 2002, An assessment of support vector machines for land cover classification. International Journal of Remote Sensing. 25, 725-749.

Karathanassi, V.; Lossifidis, C.; Rokos, D. A texture-based classification method for classifying built areas according to their density. International Journal Remote Sensing, vol. 21, p. 1807$1823,2000$.

Kuhn, M.; Johnson, K. Applied Predictive Modeling, New work: Springer, 2013.

Laliberte, A. S.; Fredrickson, E.L.; Rango, A. A comparison of three feature selection methods for object-based classification of sub-decimeter resolution Ultracam-1 imagery. International Journal of Applied Earth Observation and Geoinformation, vol.15, n. 2, p. 70-78, 2012.

Laliberte, A. S.; Goforth, M. A.; Steele, C. M.; Rango, A. Multispectral remote sensing from unmanned aircraft: image processing workflows and applications for rangeland environments. Remote Sensing. vol. 3, n. 11, p. 2529-2551, 2011a. 
Laliberte, A.S.; Rango, A. Image processing and classification procedures for analysis of sub decimeter imagery acquired with an unmanned aircraft over arid rangelands. GIScience \& Remote Sensing, vol. 48, n. 1, p. 4-23. 2011 b.

Laliberte, A.; Rango, A. Texture and scale in object-based analysis of subdecimeter resolution Unmanned Aerial Vehicle (UAV) imagery. IEEE Transactions on Geoscience and Remote Sensing, vol. 47, n. 3, p. 1-10, 2009.

Landis, J.R.; Koch, G.G. The measurement of observer agreement for categorical data. Biometrics, vol. 33, n. 1, p. 159-174, 1977.

Lechner, A. M.; Fletchera, A.; Johansen, K.; Erskine, P. Characterising Upland Swamps Using Object-Based Classification Methods And Hyper-Spatial Resolution Imagery Derived From An Unmanned Aerial Vehicle. Annals of the Photogrammetry, Remote Sensing and Spatial Information Sciences, vol. 6, p. 101-106, 2012.

Neubert, M.; Herold, H.; Meinel, G. Evaluation of Remote Sensing Image Segmentation Quality - Further Results And Concepts. Annals of the Photogrammetry, Remote Sensing and Spatial Information Sciences, p. 1-6, 2006.

Powers, R. P.; Hermosilla, T.; Coops, N. C.; Chen, G. Remote sensing and object-based techniques for mapping fine-scale industrial disturbances. International Journal of Applied Earth Observation and Geoinformation, vol. 34, p. 51 - 57, 2015.

Rossum V. G. Python tutorial, Technical Report CS-R9526, Centrum voor Wiskunde en Informatica (CWI), Amsterdam, 1995.

Quinlan, J. R. C5.0: An Informal Tutorial. Disponível em: <http://www.rulequest.com/see5unix.html>, publicado em 2013, acessado em setembro 2014.

Quinlan, J. R. Improved use of continuous attributes in C4.5. Journal of Artificial Intelligence Research, v.4, p.77-90, 1996.

R Core Team (2014). R: A language and environment for statistical computing. $R$ Foundation for Statistical Computing, Vienna, Austria. ISBN 3-900051-07-0.

Ruiz, L. F. C.; Ten Caten, A.; Dalmolin, R.S.D. Árvore de decisão e a densidade mínima de amostras no mapeamento da cobertura da terra. Ciência Rural, vol. 44, n. 6, p. 1001-1007, 2014.

Saarinen, N.; Vastaranta, M.; Vaaja, M.; Lotsari, E.; Jaakkola, A.; Kukko, A.; Kaartinen, H.; Holopainen, M.; Hyyppä, H.; Alho, P. Area-based approach for mapping and monitoring riverine vegetation using mobile laser scanning. Remote Sensing, vol. 5, n.10, p. 5285-5303, 2013.

Teodoro, A. C.; Araújo, R. Exploration of the OBIA methods available in SPRING noncommercial software to UAV data processing. SPIE Proceedings, vol. 9245, p. 1-11, 2014.

Torres-Sánchez, J; López-Granados, F.; De Castro, A.I.; Peña-Barragán, J. M. Configuration and Specifications of an Unmanned Aerial Vehicle (UAV) for Early Site Specific Weed Management. PLoS One. vol. 8, n. 3, p. 1-15, 2013.

Turner, D.; Lucieer, A.; Watson, C. An automated technique for generating georectified mosaics from ultra-high resolution unmanned aerial vehicle (UAV) imagery, based on Structure From Motion (SFM) point clouds. Remote Sensing, vol. 4, n. 5, p. 1392-1410, 2012.

Recebido em 25 de fevereiro de 2016.

Aceito em 04 de setembro de 2016. 